\title{
EXPLORING THE TRANSIENT SKY WITH THE FLY'S EYE CAMERA SYSTEM
}

\author{
L. Mészáros ${ }^{1,2}$, A. Pál ${ }^{1,2}$, G. Csépány ${ }^{1,2}$, K. Vida ${ }^{1}$, L. Kriskovics $^{1}$, and K. Oláh ${ }^{1}$
}

\begin{abstract}
To study astrophysical transit phenomena we follow an alternative strategy for getting high-cadence observations of the field. This can be achieved with our new Fly's Eye Camera System that monitors the entire sky above $30^{\circ}$ horizontal altitude. With this instrument one can observe all phenomena brighter than $\sim 15^{\mathrm{m}}$ in Sloan r-band (u', g',i' and z' filters are also available). If we stack together a few hour of images we can observe $\sim 17^{\mathrm{m}}$ faint sources. This small-sized instrument is designed for time-domain astronomy with its $150 \mathrm{sec}$ cadence. Due to the hexapod-based motion control, the instrument can be installed anywhere without any modifications, it can accomplish sky tracking automatically. These parallel robots have 6 degrees of freedom (DoF), but since any kind of rotation can be done by using only 3 DoF, the tracking with hexapods is independent from the geographical coordinates. Even polar alignment is not required, because Fly's Eye can calibrate itself based on its own observed data. The system is optimal for time-domain astronomy: detecting novae, supernovae, optical afterglows of gamma-ray bursts and other bright, fast transients, and, from the observation database such data can be obtained - even from before the discovery of the transient event. In the future when the direction of the gravitational waves will be defined precisely we will be able to detect their first multiwaveband counterparts. In addition the Fly's Eye will support the "Transient Astrophysical Objects" project which will use two new $80 \mathrm{~cm}$ robotic telescopes for follow-up observations of transients.
\end{abstract}

\section{RESUMEN}

Para estudiar fenómenos transitorios seguimos una estrategia alternativa como es la obtención de observaciones repetitivas del campo. Precisamente esto es lo que se puede lograr con un nuevo Sistema de Ojo de Mosca que puede escudriñar todo el cielo visible para alturas superiores a 30 grados. Con esta instrumentación podemos observar todos los fenómenos más brillantes de magnitud 15 en el filtro $\mathrm{r}$ de Sloan (estando también disponibles los otros filtros u, g, i, z). Sumando las imágenes durante 1 hora se puede alcanzar magnitud 17. Este instrumento concreto está diseñado para observaciones astronómicas continuadas con una cadencia de 150s. Debido al movimiento basado en un hexápodo, el instrumento puede instalarse en cualquier ubicación sin modificación alguna, de modo que pueda hacer el seguimiento celeste de manera automática. Con los 6 grados de libertad, pero usando 3 grados para cualquier rotación, el seguimiento con un hexápodo es pues, independiente de la latitud geográfica del emplazamiento. Incluso no es necesario el alineamiento polar, porque este sistema puede calibrarse a sí mismo conforme va adquiriendo los datos. Así pues, este sistema es óptimo para Astronomía de alta resolución temporal: detección de novas, supernovas, postluminiscencias ópticas de estallidos de rayos-gamma y otras fuentes transitorias rápidas y brillantes, permitiendo tener datos desde antes del fenómeno. Además, estamos considerando un proyecto complementario para usar dos telescopios de $80 \mathrm{~cm}$ de diámetro para hacer las observaciones de seguimiento.

Key Words: instrumentation: miscellaneous - techniques: photometric — telescopes

\section{INTRODUCTION}

As we spend more industrial, financial and human resources to build more and more sensitive telescopes to observe fainter and fainter objects due to saturation limits we pay less attention to brighter

\footnotetext{
${ }^{1}$ MTA Research Centre for Astronomy and Earth Sciences, Konkoly Thege Miklós út 15-17, Budapest H-1121, Hungary (meszaros.laszlo@csfk.mta.hu).

${ }^{2}$ Department of Astronomy, Eötvös Loránd University, Pázmány P. st. 1/A, Budapest H-1117, Hungary.
}

sources. Our idea was to build an instrument that covers the sky in the $9-15^{m}$ magnitude range. Furthermore we plan to design a much smaller instrument that would measure sources visible at naked eye (MANUL project; Pál et al. 2016). The Fly's Eye project started five years ago and on the third Astrorob conference we presented the design concept of the system and the assembled hexapod mount (Mészáros et al. 2014). At that time we had one camera mounted on the hexapod pointed at the zenith 
and its surrounding area. It had just been proven that the system is capable of perform a sub-pixel accurate tracking.

The low (20"/pixel) resolution was a trade-off to achieve high cadence and a very large field-of-view. The cameras are equipped with Sloan filters. In the last years we worked on software developments and upgrades. We designed the camera rack structure, the waterproof enclosure in which the environmental conditions can be controlled and a power supply cabinet for the complete instrument and dome. There are several built-in feedback subsystems (rotary encoders, limit switches, accelerometers), human monitoring (webcamera, frontend scripts that aggregate the various sensor output and network up/downlink state) and housekeeping subsystems (environmental data and power consumption sensors) to provide safe operation and to get accurate information remotely if any malfunctions occurred.

Our goal is to perform time domain astronomy. Due to it's parameters the system is capable of observing phenomena within our solar system, gain insight into star formation and evolution, extragalactic phenomena or even follow-up of brighter transient events.

Now we present the technical details of the fully assembled system, the latest results we achieved with the instrument and the upcoming tasks need to be done before it can operate as a fully autonomous telescope.

\section{HEXAPOD}

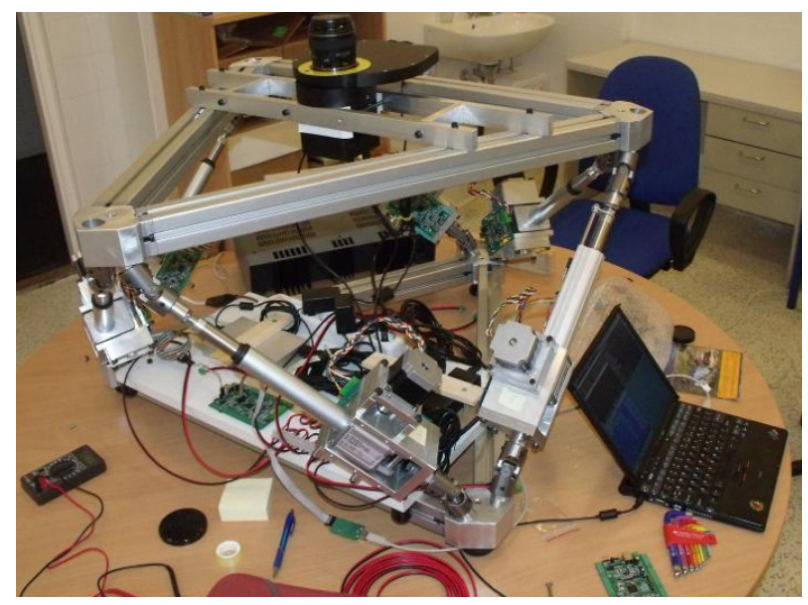

Fig. 1. The fully assembled hexapod with a single camera pointing towards the zenith is just ready for the first light test in the summer of 2013.

Using a hexapod-mount to support a camera system is unique since in astronomy these parallel robots are not so widely used (see e.g AMiBA; Chini
2000; Koch et al. 2009) and if so, these are act asthe mechanical support of secondary mirror of larger telescopes (see e.g. Geijo et al. 2006). Since this is the first time that such mount is used in direct optical imaging it was challenging to determine and design the proper parameters of the hexapod. After the first test it was proven that our concept is capable of tracking the apparent rotation of the sky with sub-pixel accuracy i.e. $1.5^{\prime \prime} \ldots 2^{\prime \prime}$ (for the optical setup see $\S 3$ ). The legs of the hexapod are linear actuators which are driven by stepper motors. The off-the-shelf actuators can be extruded or retracted with $0.05 \mu \mathrm{m}$ resolution and the maximum speed is $1 \mathrm{~mm} / \mathrm{sec}$ (see Figure 1).

Redundant feedback systems help to oversee the motion of the legs and the hexapod itself. Besides counting the (micro)steps of the stepper motors, and applying fault-tolerant limit switches, we involve absolute single-turn 12-bit magnetic rotary encoders on the actuator driving shaft where the multiple turns are counted on NVFRAM-basef ${ }^{3}$ storage. In addition, as the hexapod changes the displacement and the attitude of the payload platform, the tilt angles of the legs are also altering. We can apply our accelerometer design (Mészáros et al. 2014) to monitor these changes in the tilt angles which then provides a completely independent feedback principle.

There are several advantages of using a hexapod as a primary mount. The six leg allows the payload platform to have six degrees of freedom (DoF) with respect to the base platform. These are the lateral, longitudinal and vertical movements and the pitch, roll and yaw rotations. Since any kind of rotation can be reproduced by altering the three corresponding DoF, it means that even if three of the legs would stuck for some reason, sidereal tracking is still manageable (Pál et al. 2016). The other useful ability of the hexapod is that it is independent from the geographical location of the device since it can calibrate itself from position drifts of the observed stars. It also means that neither precise leveling nor polar alignment is required.

\subsection{Accelerometer}

The above mentioned accelerometer design is based on a microelectromechanical accelerometer system (MEMS accelerometer) which can measure dynamic or static accelerations in three directions. We use it to determine the orientation of the sensor in static local gravitational field. The chosen accelerometer has 3 -axis and it measures by a capacitive manner (see Figure 2). These commercially

\footnotetext{
${ }^{3}$ Non-Volatile Ferro-Electric Random Access Memory.
} 


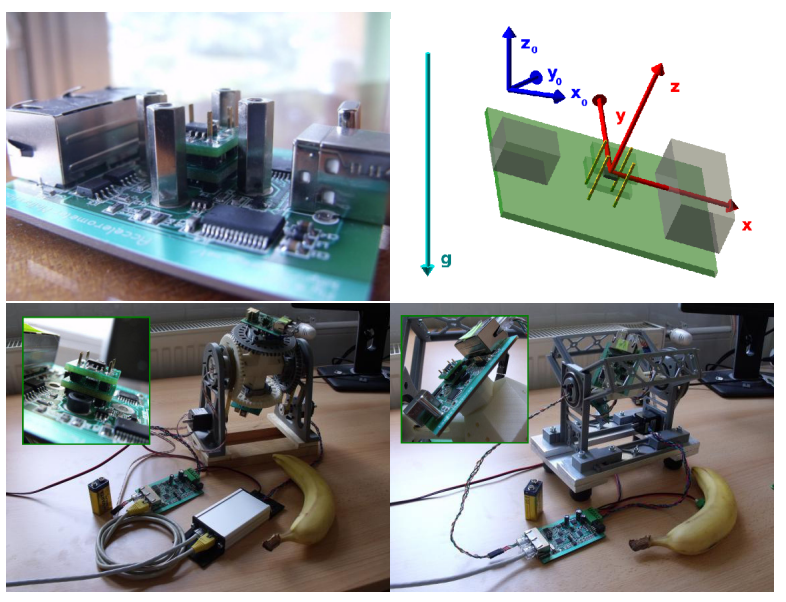

Fig. 2. Our accelerometer design. Upper left: The motherboard electronics and the stacked daughterboard system supported by the $\mathrm{I}^{2} \mathrm{C}$ bus board-to-board connectors. Upper right: A CAD model and the coordinate system of the design, showing the $\mathbf{g}$ static gravitational field as well as the various coordinate systems. Lower left: A two-axis calibration device to provide accurate calibration by exploiting the constraints between the three output channels. Lower right: A single-shaft calibration device for the calibration of the output channel values near zero acceleration.

available cheap integrated circuits come with $\sim 1^{\circ}$ absolute accuracy, however, after a proper calibration procedure this can be reduced by a factor of a one hundred, below 1' (see also Mészáros et al. 2014). Furthermore we used this technology as a feedback system for the enclosure opening/closing of the Fly's Eye (see $\S 4$ ).

\section{CAMERA UNITS}

The interchangeable camera units consist of a lens, a filter wheel, a camera, a single board computer and a supervisory electronics. We use Canon f/1.2 lenses with $85 \mathrm{~mm}$ focal length. The 5 -slot filter wheel is equipped with Sloan u', g', r', i' and z' filters. The camera itself is a $4 \mathrm{k} \times 4 \mathrm{k}$ FLI MicroLine camera with air cooled base. This setup yields a field-of-view of $26^{\circ} \times 26^{\circ}$ for each unit and yields a field-of-view of $10,000 \mathrm{deg}^{2}$ for the whole system. The overlapping of the fields is around $20 \ldots 25 \%$ with $\sim 5 \%$ overlapping three times. The resolution of this optical setup is 20 "/pixel. The 19 units cover the whole sky above $30^{\circ}$ horizontal altitude (see Figure 3$)$.

\subsection{Lens heating}

Focusing is done electrically by the supervisory electronics. We found that the focusing of the lenses is problematic in the winter cold. The lenses were not designed for such conditions thus we mounted a $6 \mathrm{~W}$ heating ring around the lenses. The ring is a series of parallel resistors covered with heat-shrink tube. The operation voltage is $12 \mathrm{~V}$ and the housing is $3 \mathrm{D}$-printed.

\subsection{Supervisory electronics}

This board is a custom designed electronics and it has the duty to reset the camera, the filter wheel and the camera controller computer (whenever it is needed, e.g. after a recovery), to monitor the power consumption of these units, to turn the lens heating on and off, and to control the lens focusing (and optionally the effective aperture stop). This board is accessible redundantly via RS232 and RS485 communication buses. Via RS232 it communicates with the single board computer and the RS485 line comes from an independent external backup bus. Furthermore, besides the internal thermal sensor, external $\mathrm{I}^{2} \mathrm{C}$-capable devices can be connected to it.

\subsection{Filter wheel issues}

In order to attain more reliable filter wheel operations, we redesigned the filter wheel control electronics and added some extra features to get feedback whether the wheel is properly set to the required slot. We also modified the housing in order to mount $0.5 \mathrm{~mm}$ optical fibers aiding the flat field calibration procedure. The idea was to direct light onto the sensor as homogeneously as possible and measure the relative sensitivity of the adjacent pixels. The source of the light is a LED, mounted on the filter wheel control electronics board. We found that the best way to project light on the sensor is if the end of the optical cables enter near to the corners perpendicularly to the sensor's edge. The test of this method and the evaluation of the results is in progress. We used the tasks of the FITSH package (Pál 2012) for image analysis.

The theoretical limit of the photometric precision of this optical setup is $\sim 4-5$ mmags, which corresponds to the photon noise level of an averagely bright $\left(\sim 10^{\mathrm{m}}\right)$ star in $\mathrm{r}^{\prime}$ band. The $\sim 15^{\mathrm{m}}$ stars are the faintest visible objects on single-exposure images of $150 \mathrm{sec}$ and this will be the saturation limit of the LSST (Ivezić et al. 2008) hence our database will be complementary for the LSST's and other all-sky surveys such as Pan-STARRS (Kaiser et al. 2002). However, by stacking up 25 consecutive frames (i.e. equivalent to a one-hour exposure), we can go down to $\sim 17^{m}$ - of course then we lost the high cadence (see Figure 4). 

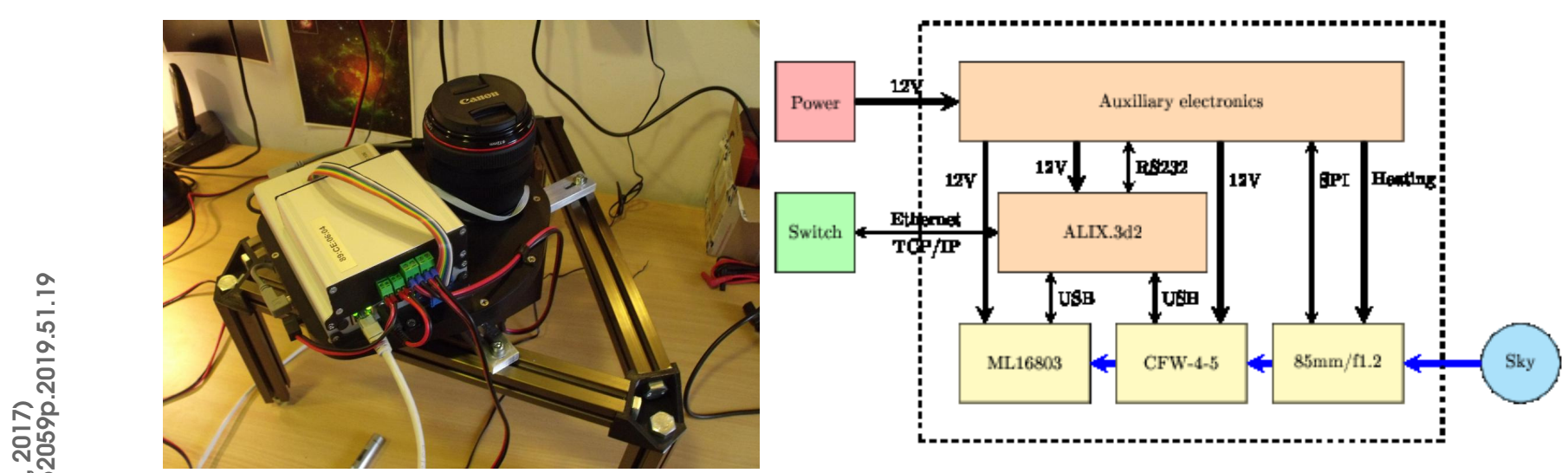

Fig. 3. Left: A complete camera node which requires a $12 \mathrm{~V}$ power supply and internet connection for primary communication. All of the other sub-unit interconnections are located within this module. An RS485 line connectes the subsequent camera nodes in a serial manner and provides a backup line if the primary TCP/IP based uplink or the embedded control computer is not accessible. Right: The block diagram of the camera unit.
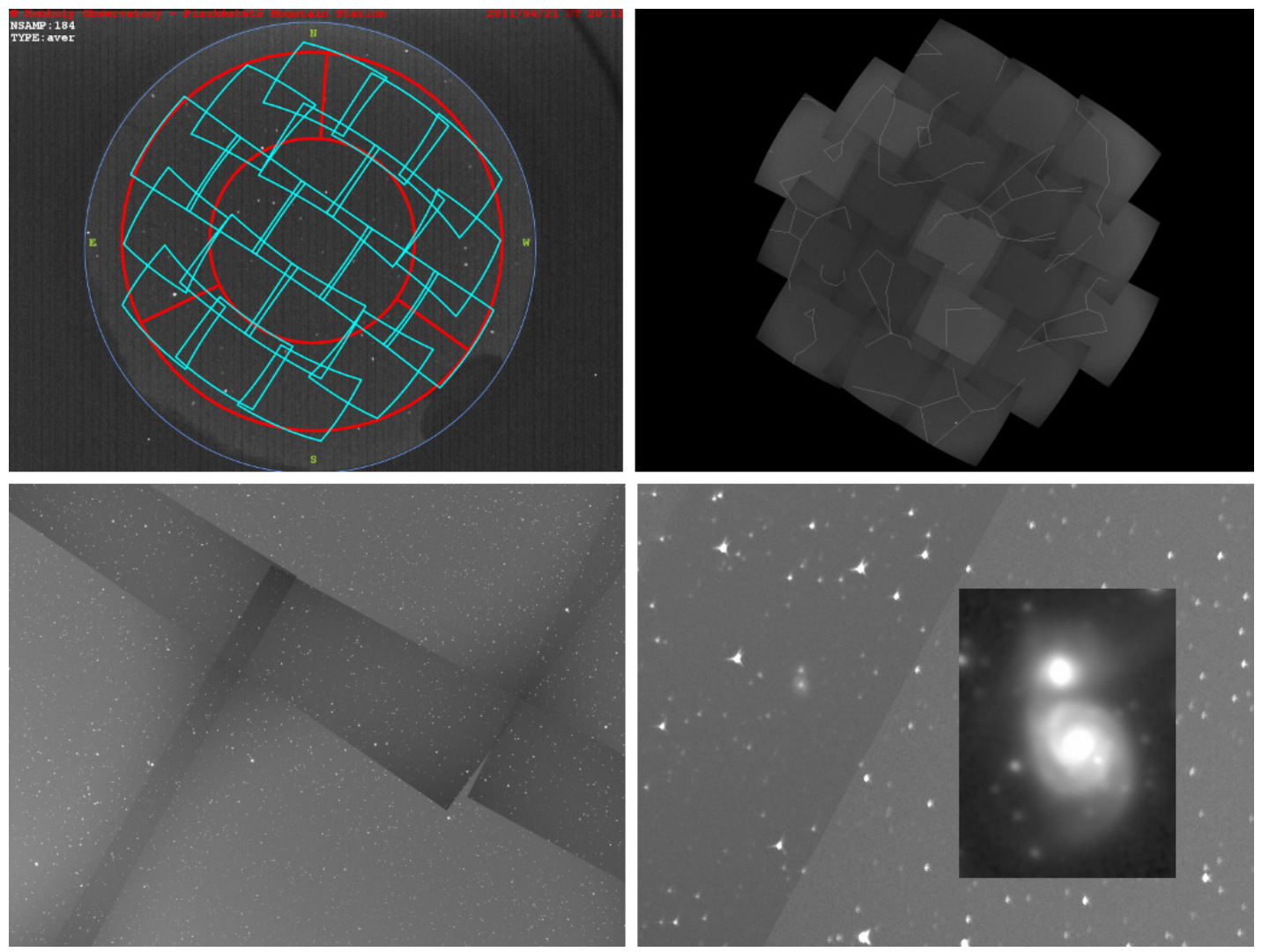

Fig. 4. Upper left: An all-sky image taken at Piszkéstető. The blue squares are the predicted field of views. Upper right: The combined field-of-view with all the cameras. All of the visible constellations are indicated. The brightness differences between the adjacent images are due to the sensitivity and bias level of the sensors which are left uncalibrated for clarity on this image. Lower left: A zoomed part of the upper-right image where the brightest stars of Big Dipper asterism can be seen/ Lower right: A more zoomed-in part of the image. The inlet image of Whirlpool-galaxy is a result of a combination of a series of images with a cumulative exposure time of 1 hour. 


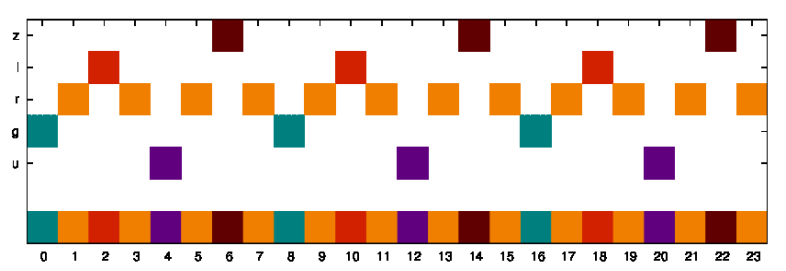

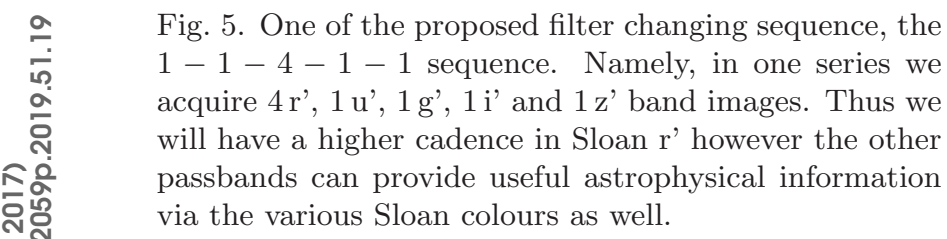

\subsection{Data acquisition scheme}

Each camera unit follows the same data acquisition scheme. The exposure time is $150 \mathrm{sec}$ with roughly $95 \%$ duty cycle. It takes $8-9 \mathrm{~s}$ to reset the hexapod, $5-6 \mathrm{~s}$ readout and $1-2 \mathrm{~s}$ changing the filters and of course these are done in parallel. The quantum efficiency of the camera is the largest in the middle of the Sloan r' band thus every second frame is taken by using Sloan r' filter. The duty cycles of the other filters are shown on Figure 5. After reading out the image the single board computer handles the uplink of the images to a computer responsible for higher level post-exposition processing and intermediate storage.

\subsection{Support structure for the 19 cameras}

The camera mount structure is a spherical shaped rack in which the camera units are installed. Although there is a $D_{3}$ symmetry, the shapes of each slots are rather versatile due to the spherical excess of the hexagonal topology. Regarding to the actual construction, the main parts of this frame are extruded aluminum profiles while the connection elements are 3 -D printed. The camera units were installed into the slots of the aluminium profiles and fixed by 3 screw with hammer nuts which can slide in the groove. Therefore, one can even adjust the orientation of the field of view in a domain of a few degrees. The structure and the installed units can be seen in Figure 6.

\section{ENCLOSURE AND POWER SUPPLY}

The Fly's Eye system is currently located at Piszkéstető Observatory, Hungary. The weather conditions are not suitable to simply leave the instrument without any protection (storms during the summer season as well as significant snowfall in winter can also occur). For protection, we designed our own enclosure.

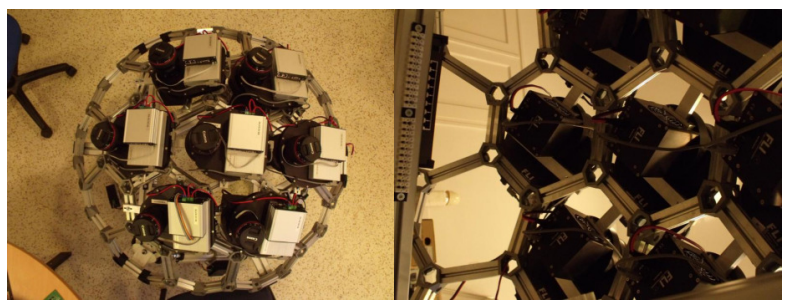

Fig. 6. Left: A partial assembly of the camera support and mount structure: 7 (out of the 19) of the camera nodes are installed into the hexagonal support structure slots. Right: An image from inside this spherical camera support structure.

\subsection{Enclosure}

In principle, the enclosure is a two-door system constructed from an extruded aluminum profile skeleton on which white-painted aluminium panels are mounted with appropriate insulation. The doors are opened/closed using outdoor linear actuators with $600 \mathrm{~mm}$ of total travel length.

To make sure that the instrument is safe, we installed several redundant monitoring systems. The actuators have a built-in Hall-sensor which sends a signal after each motor turn and the two limit switches are also provide information about the state of the actuators. We installed our accelerometer unit on both doors thus we can determine not just if the doors would stuck, but that in which position, or if they are properly closed without any gap left. Based on the aforementioned accuracy of the accelerometers (see $\S 2.1$ of Mészáros et al. 2014), the positions of the doors can well be constrained within $\sim 1 \mathrm{~mm}$. Moreover, as a "last line of defense", the whole system is monitored with a surveillance camera.

With these systems we can get reliable information about the dome enclosure. We can perform an emergency shutdown and closing if one of these would suffer breakdown and/or the sensor output states are in contradiction with each other. However we have to consider this because the failure any of these feedback subsystems does not necessarily mean that the Fly's Eye is exposed to environmental effects although this would be the safest protocol.

The environment of the inner area of the enclosure can be controlled. A $200 \mathrm{~W}$ heating cable provides a temperature to always be well above the dew point. The heat produced by the cameras during cooling would be enough to get the same output power. Two standard $80 \mathrm{~mm}$ fans (one in- one outflow) provides control over the absolute humidity. The inner surface of the enclosure is covered with $50 \mathrm{~mm}$ of polyfoam for thermal isolation. The proper closing is ensured by rubber P-shaped profiles along 

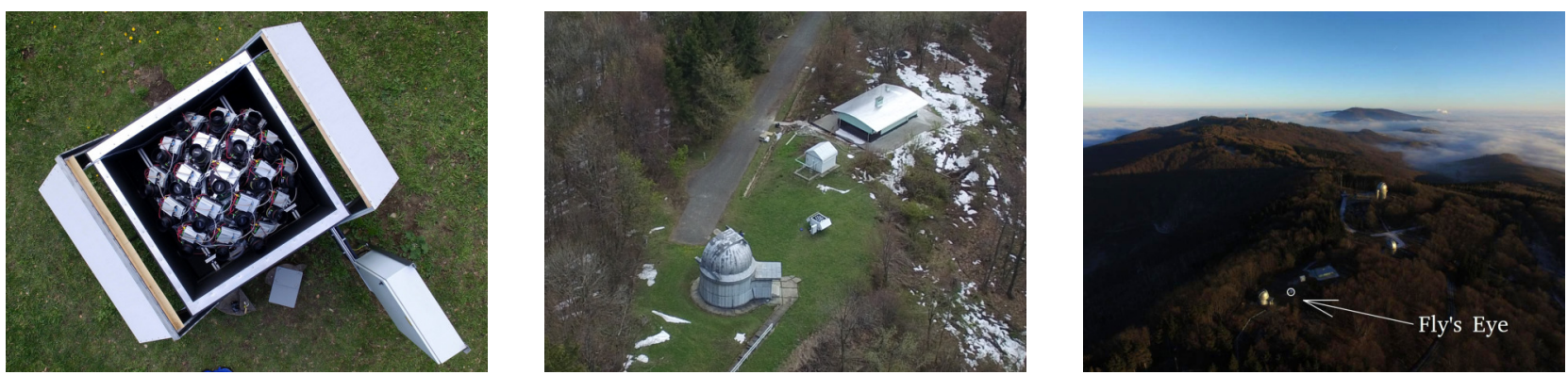

Fig. 7. Left: An aerial image of the fully assembled Fly's Eye device. The cabinet containing the power supply units and backup batteries can be seen in the lower right corner. Centre \& right: The Piszkéstető Observatory at Mátra Mountains, Hungary. The location of the device and relative size are shown by the arrow and the circle, respectively.

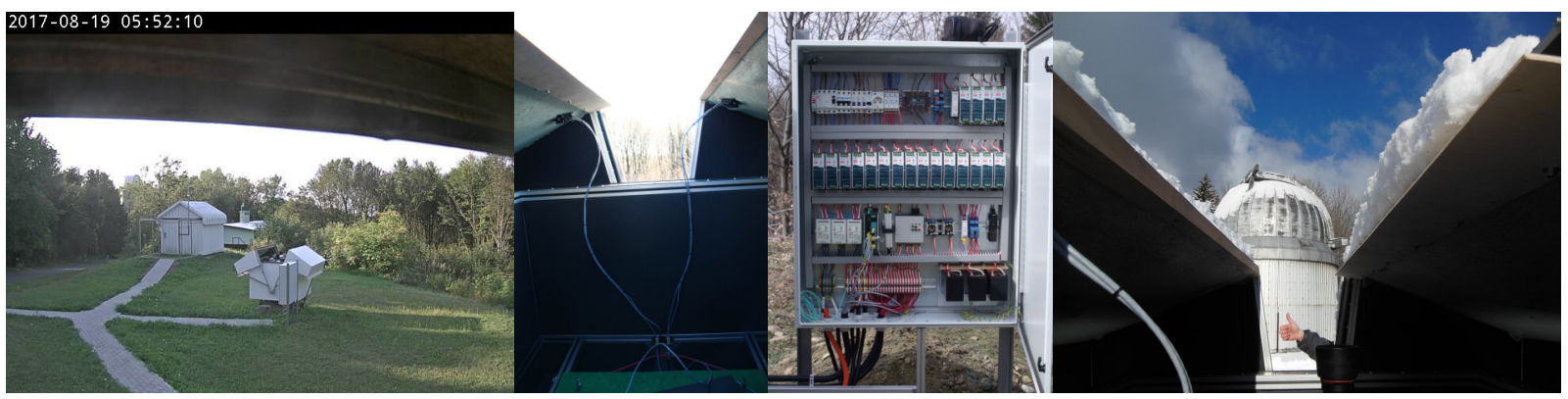

Fig. 8. $a$ : An image of the surveillance camera, showing the Fly's Eye enclosure in a partially opened state. $b$ : The accelerometers as mounted on the inner surface of the enclosure. $c$ : The cabinet containing the power supplies and the backup batteries. $d$ : During the commissioning of the enclousre, we tested how the snow accumulation can be handled by the various protective layers. After a snowstorm, the internal regions was completely dry and during the opening the accumulated snow on the top of doors slipped safely downwards.

the connecting edges. Within the enclosure, we employ environmental sensors which can measure temperature, humidity and atmospheric pressure. By installing several sensors we can measure both the spatial and the temporal fluctuation of the temperature inside the dome and, of course, such data are also used to control the aforementioned heating system.

\subsection{Power supply}

In order to avoid any mains electricity entering to the enclosure we assembled all of the necessary power supplies and backup VRLA batteries into one power cabinet which is installed right next to the the instrument enclosure itself. The state of the power supply system is also monitored continuously, including the monitoring of the individual DC voltage levels (individual $12 \mathrm{~V}$ power rails for the camera nodes, $36 \mathrm{~V}$ for the enclosure door actuators, and an additional $12 \mathrm{~V}$ rail system for the hexapod and embedded control system). The capacity of the installed VRLA batteries are sufficiently large to perform several dozens of full closing-opening cycles. All in all, by doing so we can get the hexapod to steady position and close the dome even in the case of a power cut.

Beside the actual power subsystem, this power cabinet also handles the main communication subsystem of the Fly's Eye device. Gigabit ethernet uplink is available via two redundant optical fibers that goes to a media converter switch which divide it to different directions. The communication inside the cabinet is mastered by modules that serializes TCP/IP-based commands and drives several independent RS485 bus lines in a redundant manner. Hence, all of the subsystems, including door enclosure controller, the environmental sensors or any other control and feedback units are handled and monitored via TCP/IP (see Figures 7 and 8).

\section{FINAL ASSEMBLY AND CURRENT RESULTS}

During the earlier stages of the development, we mounted only roughly the half of the camera nodes to the system (namely, 10 nodes out of the 19). The final assembly of the system with the remaining 9 units as well as a single cold-spare node has been 

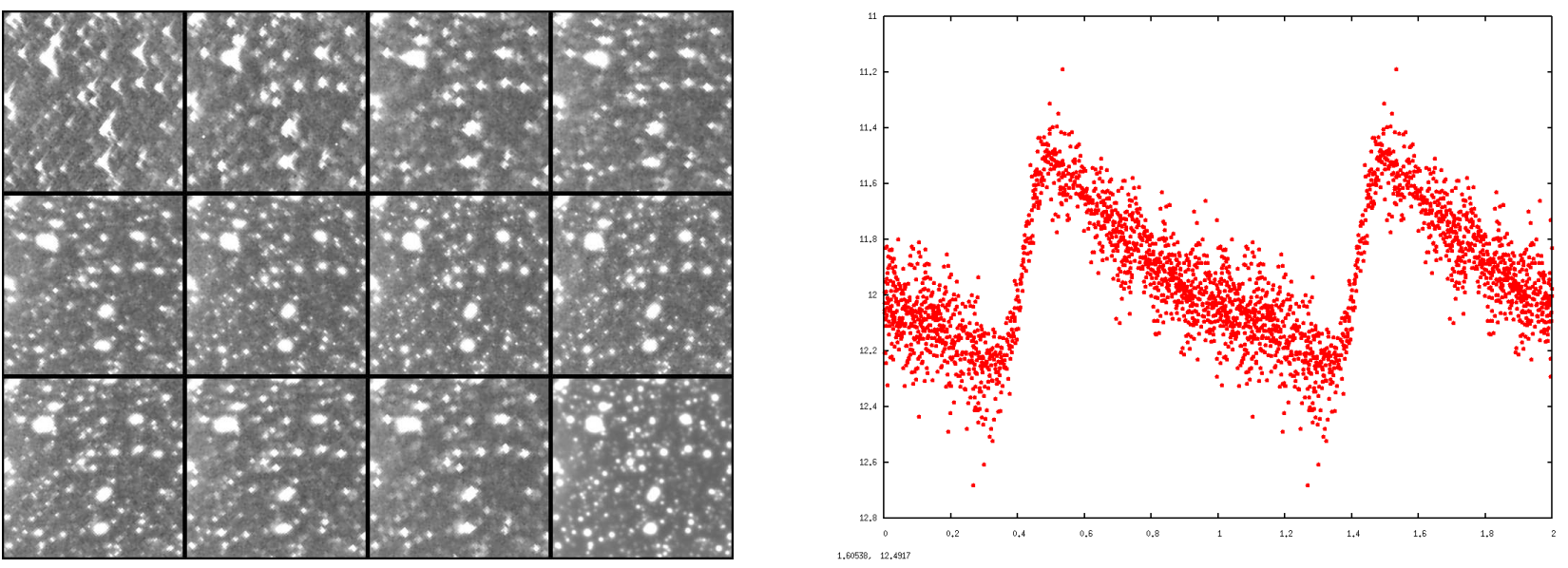

Fig. 9. Left: A series of image stamps of the same field as it moves through the field-of-view of a camera by time. The exposition of each frame is $150 \mathrm{sec}$. It can be seen that the image quality is getting better as the target area is getting closer to the centre and deteriorates as moves outward. The frame in the lower right corner is a stacked image of all of the other frames. By stacking frames we get a better quality and detailed image with a nicely averaged point-spread function due to the apparent rotation and the intrinsic dithering. Right: A raw lightcurve of an RR Lyrae star.

conducted at the end of April 2017. Since then, whenever weather conditions permit, we start observations manually after checking the weather conditions. However, no further interaction is required as the control script is capable of stopping the instrument and closing enclosure in the case of suboptimal weather conditions. We could run the instrument autonomously however there are some minor changes to be done. We also work on some optimization and improvement of the data acquisition software. Since this instrument produces $70-100 \mathrm{~GB}$ data per night we have a dedicated storage of $80 \mathrm{~TB}$ space that can handle such huge amount of data.

Currently, in parallel with the commissioning of the data acquisition we work on the data reduction pipeline. By having a large field of view we have to consider the radial optical distortion which cause an increasing shift in the position of the stars as we get farther from the image center. We created an algorithm that derive in an iterative manner the radial optical distortion via the Brown-Conrady coefficients $K_{1}$ and $K_{2}$ with respect to the gnomonic projection. We found that the values of $K_{1}=-0.0763$ and $K_{2}=-0.241$ yield a rather good astrometric accuracy in the corners as well. Defining these parameters is very important to get a good astrometric solution for the whole field. Furthermore, as the images are merged together by their sides, and if the positions are inaccurate, the merging of the overlapping areas, and hence the derivation of the photometric magnitudes will fail. These algorithms will be available for the public in the next release of the FITSH package (Pál 2012) (see Figures 9 and 10).

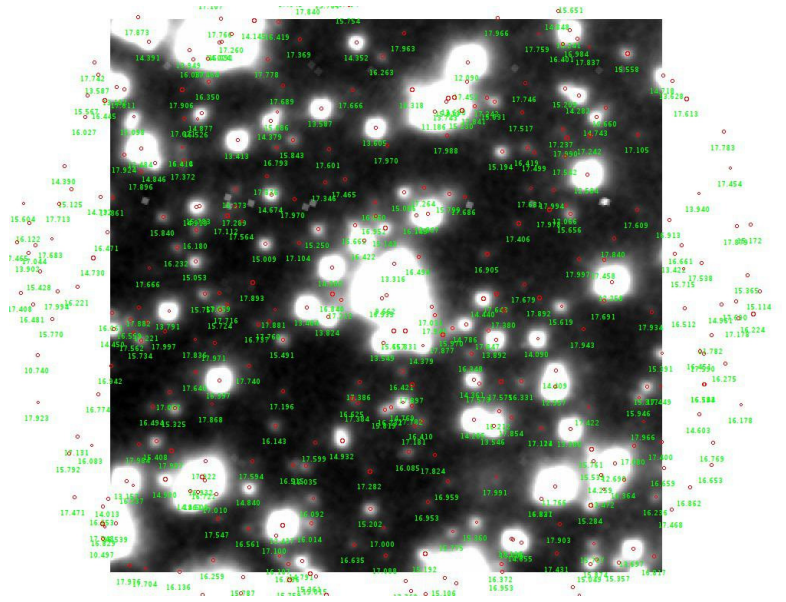

Fig. 10. A stacked image combined from 25 individual frames, equivalent with a single image taken with an exposure time of 1 hour. The green markings indicate the magnitude values of the identified stars. It can be seen that the faintest sources have a brightness of $17^{\mathrm{m}}$ or below, slightly depending on the actual colour of the star.

Acknowledgements: The development and the building of this instrument is supported by the Hungarian Academy of Sciences via the grant LP2012-31. The operation is supported by the GINOP-2.3.2-152016-00033 project which is funded by the Hungarian National Research, Development and Innovation Fund together with the European Union. We thank 
H. Deeg for the useful discussions and the possibility for relocation our device to the intended location of the PASS project (Deeg et al. 2004). Additional support is also received via the OTKA grants K-109276, K-104607 and K-113117. Krisztián Vida is supported by the Bolyai János Research Scholarship of the Hungarian Academy of Science. In our project, we involved numerous free \& open source software, including gEDA (for schematics and PCB design), FreeCAD (3D designs), CURA (3D slicing, GCODE generation and printing control) and AVRGCC (for MCU programming).

\section{REFERENCES}

Chini, R. 2000, RVMA, 13, 257

Deeg, H. J., et al. 2004, PASP, 116, 985

Gehrels, N., et al. 2004, ApJ, 611, 1005

Geijo, E. M., et al. 2006, SPIE, 6273, 38

Ivezić, Ž., et al. 2008, arXiv:0805.2366

Kaiser, N., et al. 2002, SPIE, 4836, 154

Koch, P. M., et al. 2009, ApJ, 694, 1670

Mészáros, L., et al. 2014, PASP, 126, 769

Mészáros, L., et al. 2014, RMxAC, 45, 94

Pál, A. 2012, MNRAS, 421, 1825

Pál, A., et al. 2016, SPIE, 9908, 59

Pál, A., et al. 2016, PASP, 128, 962 